\title{
Aldo-ketoreductase 1 (AKR1) improves seed longevity in tobacco and rice by detoxifying reactive cytotoxic compounds generated during ageing
}

Kodadinne Narayana Nisarga ${ }^{\dagger}$, Ramu S Vemanna ${ }^{\dagger}$, Babitha Kodekallu Chandrashekar, Hanumantha Rao, Amaranatha Reddy Vennapusa, Ashwini Narasimaha, Udayakumar Makarla* and Mohan Raju Basavaiah

\begin{abstract}
Background: Maintenance of seed viability is an important factor for seedling vigour and plant establishment. Lipid peroxidation mediated reactive carbonyl compounds (RCC's) and non-enzymatic modifications of proteins through Maillard and Amadori products reduce seed viability and seedling vigour.

Results: In this study, the relevance of RCCs on genotypic variation in rice seed viability and overexpression of an aldo-ketoreductase (AKR1) enzyme that detoxify cytotoxic compounds to improve seed viability and vigour was studied. Physiological and biochemical approaches were integrated to quantify the variation in seed viability and seedling vigour in rice genotypes after exposing to ageing treatment. AKR1 was overexpressed in a susceptible rice genotype and tobacco to study the relevance of reduced RCC's on seed viability and seedling vigour. The glycation and lipid peroxidation compounds accumulated after accelerated ageing treatments in rice genotypes. The accumulation of malondialdehyde, methyl glyoxal, Maillard and Amadori products affected the seed viability and germination as they showed a significant negative relationship. The transgenic rice and tobacco seeds expressing AKR1 showed lower levels of cytotoxic compounds and glycation products that resulted in improved seed viability and seedling vigour in rice and tobacco.

Conclusions: The study demonstrates that, reactive cytotoxic compounds affect the seed viability during storage. Detoxification of reactive cytotoxic compounds by Aldo-keto reductases is one of the mechanisms to improve the seed longevity during storage.
\end{abstract}

Keywords: Oxidative stress, Seed viability, Protein glycation, Ageing, Carbonyl stress, Transgenics

\section{Background}

Seeds are mysterious genetic capsules of plant life cycle essential for establishment and reproduction to maintain the species from generation to generation. For the crop establishment in the field, viable seeds are crucial input. Good quality seeds of improved varieties can contribute to about $20-25 \%$ increase in productivity (Hasanuzzaman, 2015). Therefore, there is a need to sustain seed

\footnotetext{
* Correspondence: udayakumar_m@yahoo.com

${ }^{\dagger}$ Equal contributors

Department of Crop Physiology, University of Agriculture Sciences, GKVK, Bengaluru 560065, India
}

viability during storage and improve the seedling vigour. During ageing process, seeds deteriorate, lose quality, viability and vigour especially under high temperature and humidity (Kapoor et al., 2011). Under increased moisture conditions, temperature and humidity, loss of seed viability is due to enzymatic and non-enzymatic modification of proteins by Amadori and Maillard reactions (Murthy and Sun, 2000; Miyata et al., 2000). Seed deterioration has shown to be influenced by a number of factors related to seed structure and biochemical characteristics (Shaban, 2013). Increased lipid peroxidation leading to loss of membrane integrity, reduced energy metabolism, impairment of RNA and protein synthesis, 
formation of protein iso-aspartyl residues, DNA degradation, accumulation of reactive oxygen species (ROS) and reactive carbonyl compounds (RCCs) which enhances seed deterioration during storage (Mudgett and Clarke, 1993; Mudgett et al., 1997; Murthy et al., 2003; Kibinza et al., 2006; Mohammadi et al., 2011; Mano, 2012; Shaban, 2013).

Lipid peroxidation is the major cause for seed deterioration that damage integrity of cell membrane (Gidrol et al., 1989). Lipoxygenases catalyze the oxidation of polyunsaturated fatty acids to produce conjugated unsaturated fatty acid hydroperoxides (Feussner and Wasternack, 2002). Lipid degradation caused by phospholipase D (PLD) activity is known to be responsible for seed deterioration in Arabidopsis (Wang et al., 2012). In rice, transcript levels of OsPLD 1 and $\alpha 3$ increased during ageing. Accumulation of lipid peroxides lead to the production of reactive carbonyl compounds (RCCs) that are $\alpha, \beta$-unsaturated aldehydes and ketones. These RCS include melondialdehyde (MDA), acrolein, 4-Hydroxy(E)-2-nonenal (HNE) and 4-Hydroxy-(E)-2-hexenal (HHE) are most reactive and toxic that diffuse across the membrane with longer half-life and modify the proteins and nucleic acids (Sayre et al., 2006; Mano, 2012). Oxidative stress is the primary cause to produce many long lived cytotoxic RCCs that form aggregates with proteins and lipids that generates advanced glycation end products (AGEs) and advanced lipoxidation end products (ALEs) which affect metabolic pathways (Ott et al., 2014; Semchyshyn, 2014).

Genetic enhancement studies for improving seed viability and seedling vigour have identified several genes that are involved in regulating seed deterioration. Overexpression of PIMT1 in Arabidopsis reduced the accumulation of L-isoaspartyl residues in seed proteome and showed increased germination and seedling vigour (Oge et al., 2008). Transgenic Arabidopsis seeds overexpressing lotus (Nelumbonucifera L.) NnMT2a and NnMT3 (metal-binding protein metallothionein) genes showed an increased resistance to accelerated ageing treatment and enhanced seedling vigour (Zhou et al., 2012). DNA repair genes act during the early phase of germination and expression of DNA ligase-I showed improved seed vigour in Arabidopsis (Andreuzza et al., 2010; Ventura et al., 2012). A forward genetic approach used to validate seed longevity trait based on activation-tagging mutants lead to the identification of an RSL1a ubiquitin ligase that showed enhanced seed longevity (Bueso et al., 2014).

During ageing, accumulation of lipid peroxidation compounds and RCC's reduces seed viability and therefore, detoxification of these cytotoxic compounds may enhance seed viability and vigour. Aldehyde reductase from Mung bean belonging to Aldo-keto reductases (AKRs) family has been reported to detoxify a wide variety of lipid peroxidation or glycolysis derived cytotoxic compounds (Colrat et al., 1999). The AKRs catalyze a variety of carbonyl compounds including aldehyde form of glucose that is reduced to corresponding sugar alcohols and sorbitol (Sree et al., 2000). From this context, the focus of this study is to understand the role of cytotoxic compounds that accumulate during seed ageing and the relevance of detoxifying enzymes in seed deterioration in rice. The study demonstrates that, accumulation of RCCs during seed storage cause seed deterioration. Overexpression of RCC's detoxifying enzyme, Pseudomonas AKR1 (PSAKR1) in tobacco and rice showed improved seed viability by reducing the levels of MDA, methyl glyoxal (MG), Maillard products and Amadori compounds under controlled accelerated deterioration conditions and improved seed viability and seedling vigour.

\section{Results}

\section{High temperature and humidity during seed storage} affect seed viability in rice genotypes

To assess the variability in seed viability, the rice genotypes were exposed to accelerated ageing (AA) treatment for 4,6 and 8 days at $45^{\circ} \mathrm{C}$ with $100 \%$ relative humidity. All genotypes germinated after 4 days of ageing and after 8 days only two genotypes AC39020 and AC35310 showed germination. To assess differential response of the genotypes, 6 days of AA was found to be optimum and genotypes showed significant variability in seedling vigour index and germination percentage. TTC assay was carried out as a measure of dehydrogenase activity and is an indirect measure of seed viability status. TTC reduction was higher in genotypes AC39018 and AC35313 while it was less in Tellahamsa and MAS (Fig. 1a \& b). Further the extent of loss of membrane damage was negatively related to seed viability. The $\mathrm{pH}$ of the seed leachate also showed a negative relationship with seed viability (Fig. 1c \& d). The data suggests that under ageing process rice seeds undergo many biochemical changes and hence deteriorates faster and results in loss of viability.

\section{Accumulation of RCCs affects germination and seedling vigour in rice genotypes under accelerated ageing conditions}

The variability in the accumulation of RCC's and their role in seed germination were assessed in the seeds of rice genotypes exposed to accelerated ageing. Seeds subjected to accelerated ageing showed reduced germination and seedling vigour (Fig. 2a). The genotypes MAS and Tellahamsa showed less than 50\% germination compared to IET16348 and KMP-175 genotypes that showed higher germination percentage (Fig. 2b). Further, the seedling vigour was also found to be significantly low in MAS and Tellahamsa. MAS, Tellahamsa and IET-16348 


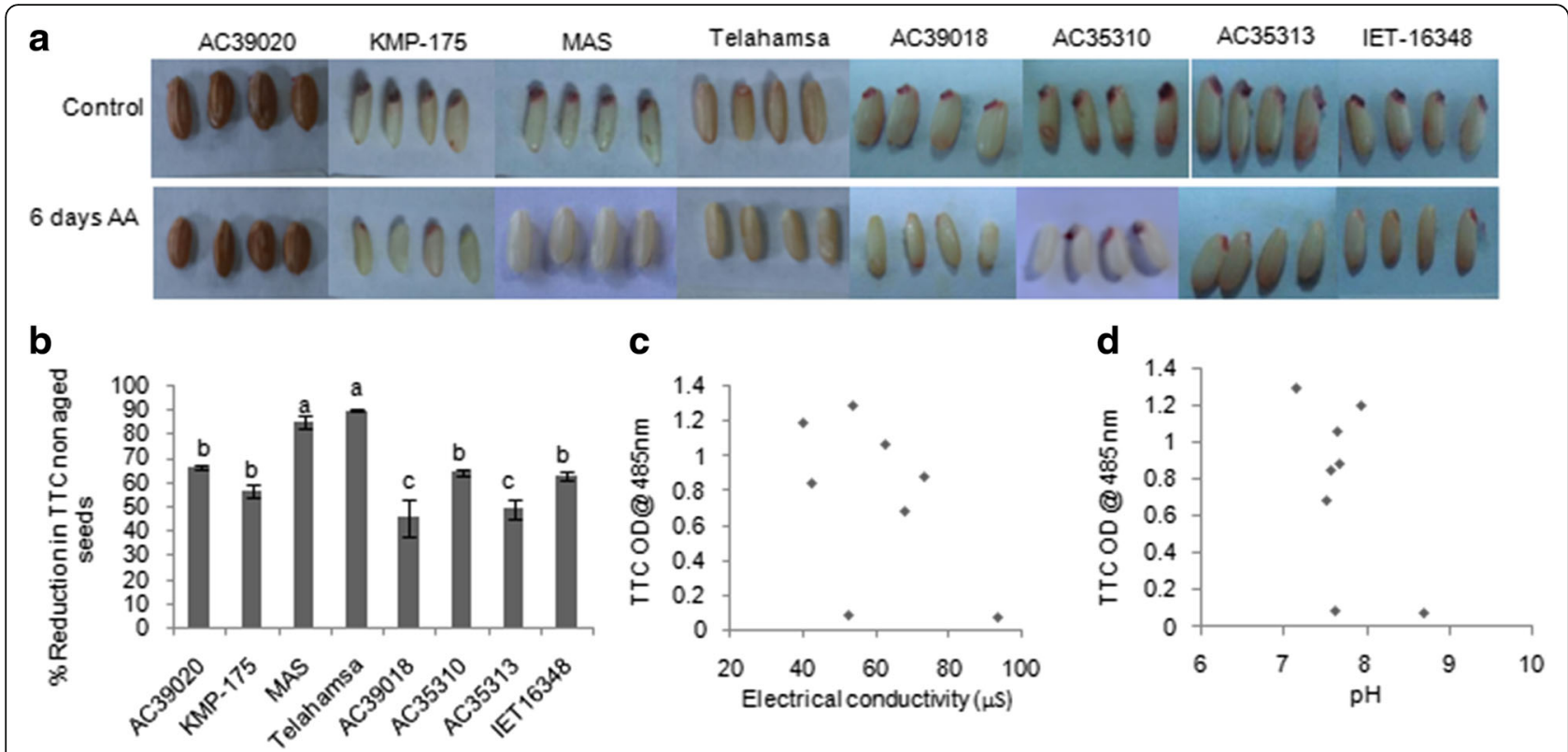

Fig. 1 Variations in seed viability across rice genotypes under aged and non-aged conditions. a Photograph showing TTC staining of rice genotypes showing variation in seed viability under control and accelerated ageing conditions. The photographs were taken after one day of incubation in TTC stain. $\mathbf{b}$ Variability in dehydrogenase activity as quantified by reduction in TTC levels over control showing differences in seed viability, $(n=10)$ (Alphabets with same letters are non-significant at $p=<0.05$ ). c Relationship between seed viability (an indication of $T$ C reduction) and membrane damage in rice upon ageing treatment and $\mathbf{d}$ Effect of pH on dehydrogenase activity (as indicated by $\Pi \mathrm{TC}$ reduction) in rice seeds upon ageing treatment

showed very less seedling vigour index compared to other genotypes (Fig. 2a \& c). The germination speed index (GSI) an indication of time taken for seed germination was less in all the genotypes except Tellahamsa that had 8 GSI (Additional file 1: Figure S1a). The seed germination and seedling vigour showed a significant positive correlation with seed viability as measured by levels of TTC assay as a reflection of NADPH dependent dehydrogenase activity (Fig. 2d \& e). Further, significantly higher accumulation of cytotoxic compounds was observed in aged seedlings. MG accumulated in seeds upon ageing treatment showed an inverse correlation seed germination (Fig. 2f). Similarly, Maillard and Amadori products, had similar effect on seed germination indicating that cytotoxic compounds affect the seed germination and seedling vigour (Fig. 2g). Amongst the genotypes, Tellahamsa and AC35313 showed higher levels of MG compared to the other genotypes (Additional file 1: Figure S1b). The increase in Maillard products was significantly higher in Tellahamsa genotype during ageing conditions (Additional file 1: Figure S1c). However, Amadori products (glycation end products) accumulated significantly higher levels in genotypes such as AC39020, AC39018 and AC35313 under ageing condition (Additional file 1: Figure S1d). Accumulation of Amadori products negatively correlated with germination percentage that affected the seedling vigour (Fig. 2g). Further, $\alpha$ amylase enzyme activity was significantly increased in aged seedlings specifically in the genotypes AC39020 and KMP175 (Additional file 1: Figure S1 e \& f). These genotypes also showed higher accumulation of total sugars under ageing condition (Additional file 1: Figure S1f). The results clearly demonstrate that RCC's accumulate during ageing and negatively influence on seed vigour.

\section{Overexpression of PsAKR1 gene in tobacco detoxifies RCCs and improves seed viability and germination}

In rice genotypes, reduced seed viability and germination was correlated with increased accumulation of RCCs suggesting that managing these compounds is crucial to improve seed viability, germination and vigour. In view of this, to reduce the accumulation of RCC's in seeds one of the RCC detoxifying enzymes, Aldoketoreductase-1 from Pseudomonas strain (PsAKR1) that was codon optimized to plants and overexpressed in tobacco. The stable differentially expressed transgenics lines (Additional file 1: Figure S2a) of T3 generation were used to study the seed viability and vigour by exposing to accelerated ageing treatment for 48 and $72 \mathrm{~h}$. The transgenic seeds after accelerated ageing showed higher levels of TTC reduction suggesting sustained NADPH dependent dehydrogenases activity compared to wild type seeds (Fig. 3a \& b). Under normal conditions, transgenic seeds showed early germination than the wild type seeds. After $48 \mathrm{~h}$ of ageing treatment, transgenic seeds showed $\sim 80 \%$ germination whereas, wild type seeds 


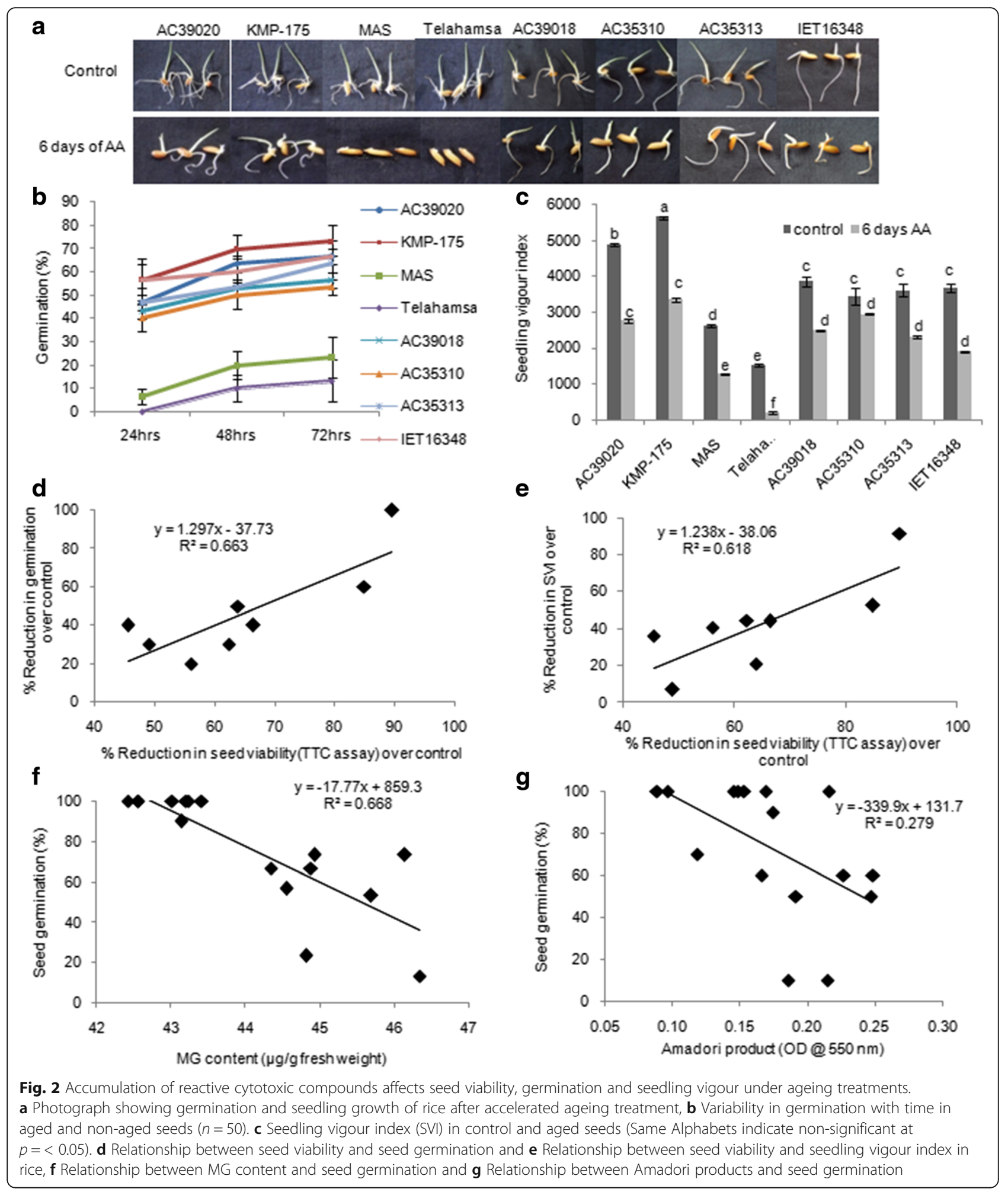

showed $65 \%$ germination. Even after $72 \mathrm{~h}$ of ageing transgenic seeds showed more than $50 \%$ seed germination whereas wild type seeds showed less than $10 \%$ germination (Fig. 3c \& d). The germination percent of transgenic seeds was positively correlated with higher dehydrogenase activity (Fig. 3e). Further, the accumulation of MDA and MG was less in transgenic seeds compared to wild type seeds (Additional file 1: Figure S2b \& c) and the levels of these compounds negatively associated with the seed germination (Fig. $3 f$ \& g). Even the accumulation of Amadori 
and Maillard products under ageing conditions was significantly low in transgenic plants (Additional file 1: Figure S2d \& e). The low level of RCCs including AGEs in tobacco transgenics indicates efficient detoxification of RCCs by PsAKR1 (Fig. 3).

\section{Less viable and susceptible rice genotype (Tellahamsa) under ageing condition was rescued by overexpression of PsAKR1}

To improve the seed viability of susceptible rice genotype Tellahamsa, transgenic plants expressing AKR1 were developed and stable lines were identified by screening on glyphosate as described in our previous study (Vemanna et al., 2016). The transgenic plants were confirmed for presence of transgene using genomic DNA PCR (Additional file 1: Figure S3a). Further based on the expression profile four transgenic lines with differential transcript levels were selected to assess the seed viability and seedling vigour (Fig. 4a). Transgenic rice seeds upon ageing treatment showed higher seed viability that was reflected in improved seed germination compared to wild type seeds. The transgenic seeds showed higher per cent germination than the wild type seeds under normal as well as ageing conditions (Fig. 4b \& c). Further the AKR1 activity in transgenic seeds was significantly higher compared to wild type seeds (Fig. 4d). Under ageing conditions the AKR1 activity was reduced even in transgenics, however the transgenic seeds still maintained adequate activity to maintain the seed viability (Fig. 4d). The seedling vigour index was also higher in all the transgenics compared to wild type plants (Fig. 5a). Further, the transgenic seeds had significantly less electrolyte leakage than wild type seeds (Additional file 1: Figure S3b). The $\mathrm{pH}$ of the seed leachate was less acidic in transgenics whereas wild type seed leachate had alkaline $\mathrm{pH}$ (Additional file 1: Figure S3c). The EC and $\mathrm{pH}$ were negatively correlated with seed germination and seedling vigour index (Fig. 5b \& c). Similarly the levels of MG is negatively influenced on seedling vigour index (Fig. 5d). The transgenic seeds under ageing treatment showed significantly reduced levels of MG and MDA (Fig. 6a \& Additional file 1: Figure S3d) and therefore, a significant negative relationship was observed between cytotoxic compounds and seed germination (Fig. 5e \& f).

\section{The AKR1 transgenic seeds detoxify the cytotoxic MG to improve seed viability and vigour}

The transgenic rice seeds expressing AKR1 showed reduced MG levels and the increasing activity in the transgenic lines corelates with the reduced MG levles both in controlled and ageing treatments (Fig. 6b). Further, to assess whether overexpression of AKR1 in susceptible rice genotype Tellahamsa rescues the seedlings from MG induced effect, the growth response of germinated seedlings was assessed at $10 \mathrm{mM}$ and $20 \mathrm{mM}$ MG. In both the concentrations of MG, the transgenic seedlings had maintained higher growth and shoot growth was less affected compared to wild type seedlings (Fig. 6c \& d). Higher AKR activity in transgenic seeds detoxify the MG and enhances the seed viability and vigour. These results demonstrated that, detoxifying the RCCs that are accumulated during ageing conditions by AKR1, improve seed viability and seedling vigour. Therefore overexpression of PsAKR1in Tellahamsa genotype rescued the susceptible phenotype during ageing process.

\section{Discussion}

Seed viability and seedling vigour has phenomenal relevance in crop establishment and productivity especially under abiotic stress conditions. However, during storage, seed viability reduces rapidly with the accumulation of many cytotoxic compounds. Seeds deteriorate because of high temperature and relative humidity $(\mathrm{RH})$ leading to oxidative damage to lipids, proteins and nucleic acids affecting cellular metabolic activities (Mano, 2012). Oxidative damage specifically on lipids produces peroxidation compounds like MDA and MG that are highly reactive electrophiles. These compounds further acts on proteins through Maillard reaction or Schiff's bases to form Amadori products that leads to the production of AGE's and ALE's (Miyata et al., 2000). From this context, managing RCC's have greater relevance in improving the seed viability. It was reported that, different eco-geographic regions are responsible for the variation in japonica cultivars having low longevity than upland cultivars (Rao and Jackson 1996). The genotypic variation in indica rice genotypes that are tested in this study showed variability in seed viability under ageing conditions. The accumulation of reactive carbonyl compounds in rice genotypes exposed to seed ageing showed genotypic variability and it was related to seed germination. The rice genotypes Tellahamsa and MAS showed early loss of seed viability and germination mainly due to accumulation of cytotoxic compounds. The reduced dehydrogenase activity was tightly linked with seed viability. The susceptible and less viable genotypes such as Tellahamsa and MAS showed distinctly low dehydrogenase activity and membrane stability compared to rest of the genotypes. The accumulation of Maillard and Amadori products signifies these two genotypes accumulated more AGEs and ALEs under ageing conditions. The Maillard reaction product has been shown to impair protein function leading to browning reaction and thus affecting the metabolic activity (Wettlaufer and Leopold 1991). The results from genetic variability in studies suggest that, RCC accumulation significantly affects the seed viability in rice. 


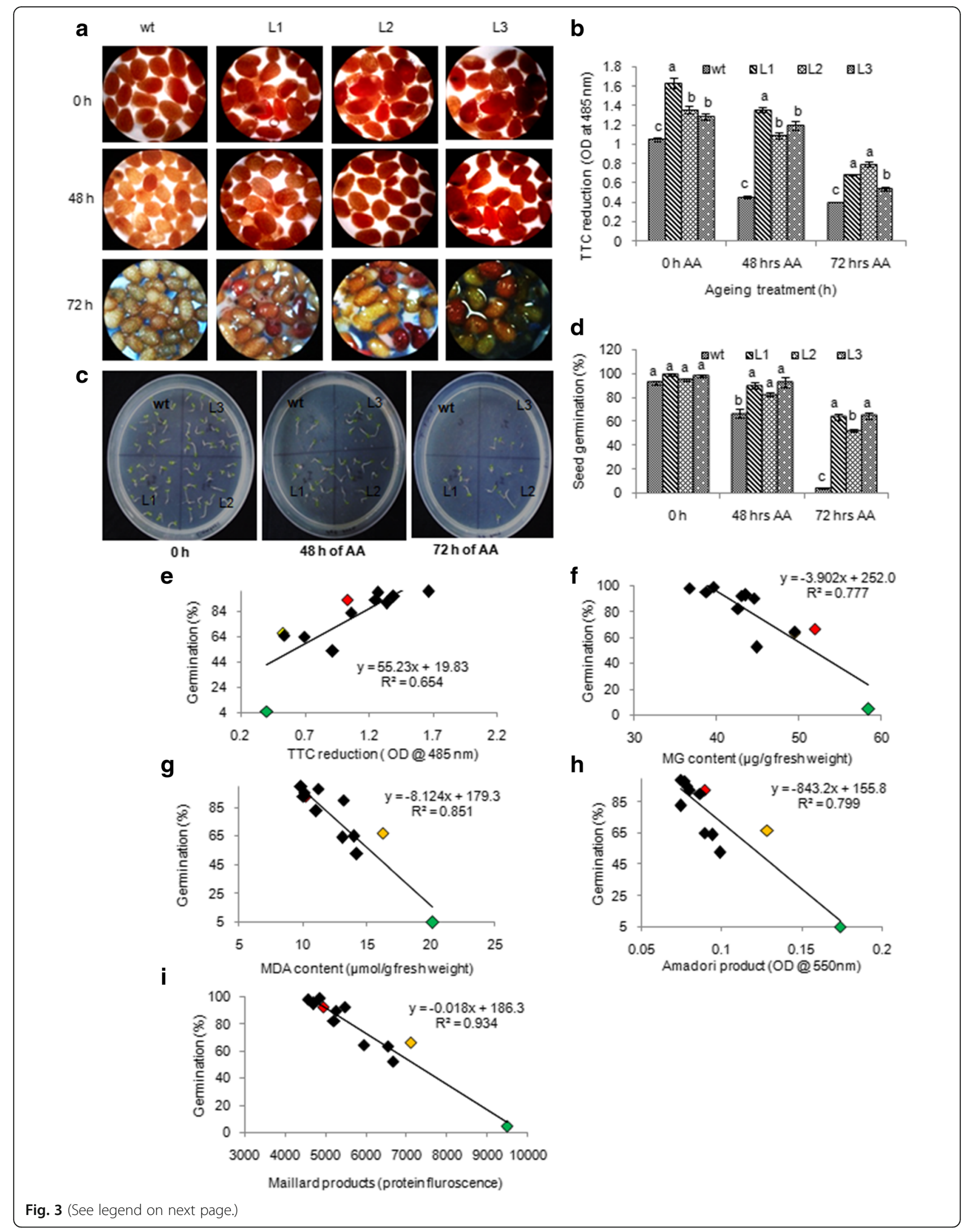


(See figure on previous page.)

Fig. 3 PSAKR1 expressing tobacco transgenic seeds showed improved seed vigour and reduced reactive carbonyl compounds, a Transgenic seeds showing more TTC staining than wild type seeds, $\mathbf{b}$ Extent of TTC reduction (an indication of seed viability in WT and transgenic seeds at different durations of ageing treatment, $\mathbf{c}$ Differential seed germination of WT and transgenic seeds, $\mathbf{d}$ Germination percentage of transgenic and wild type seeds after accelerated ageing treatments, error bar indicate mean values from three biological replicates that are having 10 seeds each. $t$-test for both wildtype and transgenic seeds were conducted. Alphabets with same letters are non-significant at $p=<0.05$. e, $\mathbf{f} \mathbf{g}, \mathbf{h}$ and $\mathbf{i}$ Correlations between germination \% with TTC, methylgloxal (MG) content, malondialdehyde (MDA) content, Amadori product and Maillard products. Data points in colour indicate different time points of wild type seeds exposed to aging conditions at control (red), $48 \mathrm{~h}$ (yellow) and $72 \mathrm{~h}$ (green)

The enhanced level of $\alpha$-amylase activity in all the rice genotypes when exposed to higher temperature during ageing treatment was evident with the accumulation of higher levels of total sugars. However, a significant variation in the genotypes was observed. Increased rate of germination at higher temperature is mainly attributed to enhanced respiration with the accumulation of reducing sugars. The $\alpha$-amylase activity during germination is considered as a biomarker in several species (Koning, 1994). In this study, the increased $\alpha$-amylase activity resulted in accumulation of more sugars in rice genotypes under ageing conditions. However, in spite of higher $\alpha-$ amylase activity, rice genotypes did not show increased germination. Instead, our results showed that the increased sugar content in Tellahamsa and MAS was negatively correlated with germination percentage. The increased $\alpha$-amylase activity during ageing leads to accumulation of sugars and their utilization in respiratory metabolism largely depends on the activity of dehydrogenases associated with citric acid cycle. During AA, the dehydrogenase activity was reduced, that may have resulted in the conversion of glucose into MG. During photosynthesis $\mathrm{CO}_{2}$ converts in to sugars, and during respiration auto oxidation of sugars is inevitable and produces ROS and sugar derived RCCs (Takagi et al., 2014). In microbes the enzymatic conversion of dihydroxyacetone phosphate catalysed by MG synthase produces MG (Richard, 1984). The MG, Maillard and Amadori products thus produced during AA conditions affected the cellular enzymes thus affecting the metabolic activity. The higher level of RCC's under ageing conditions observed in this study seems to be the major contributing factor for loss of seed viability and vigour in rice genotypes. An inverse relationship between cytotoxic compounds and seed viability was observed in several crop species (Murthy et al., 2003). In soybean, increase in Maillard reaction during accelerated ageing caused enhanced seed deterioration (Sun and Leopold 1995).
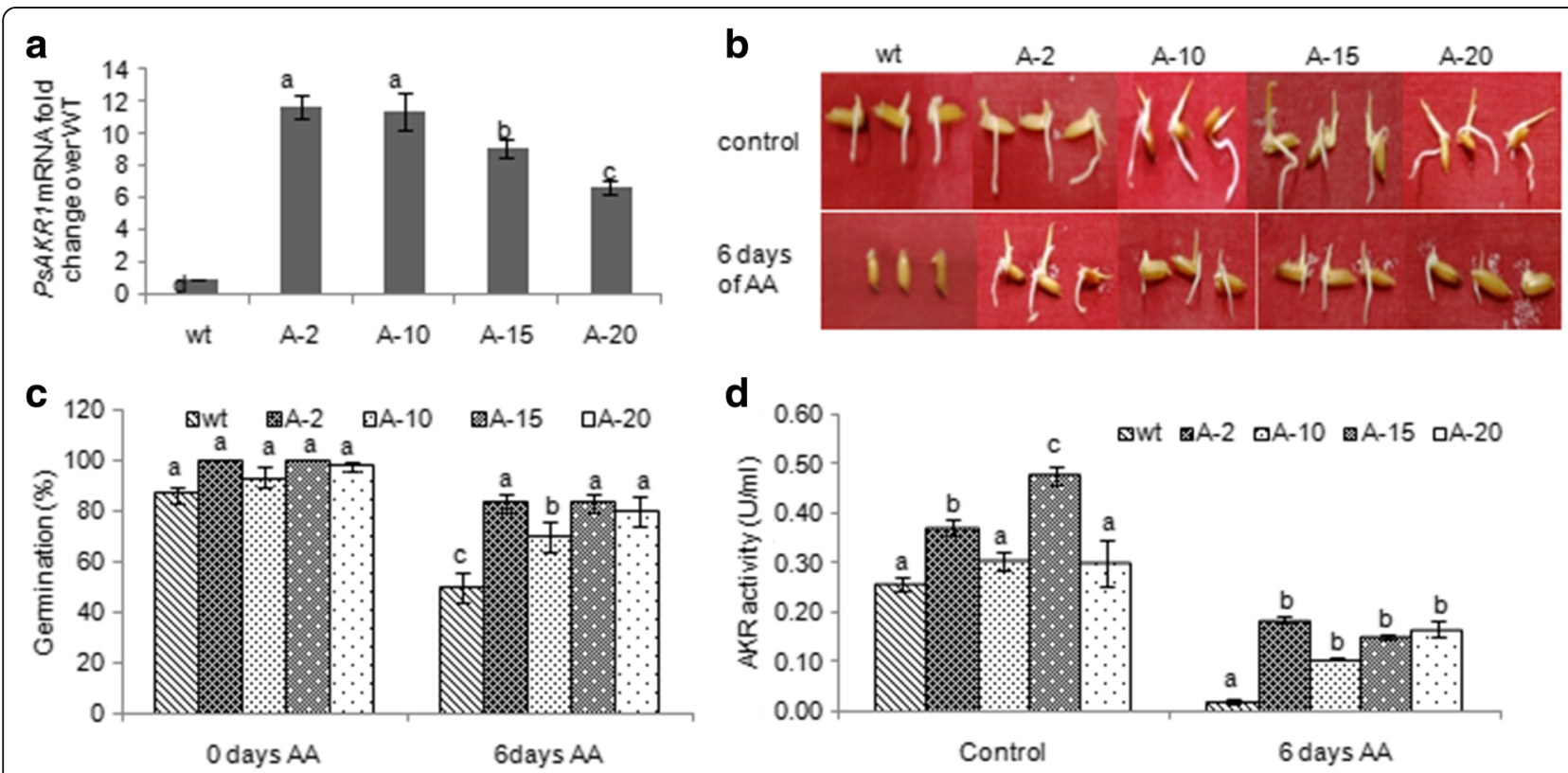

Fig. 4 Overexpression of PSAKR1 in susceptible rice genotype Tellahamsa showed improved seed viability and germination. a Transcript levels of PSAKR1 in different transgenic rice lines, The RNA from seeds were isolated and the cDNA is used as a template, $\mathbf{b}$ Photograph of transgenic rice seeds showing early germination after ageing treatment, c Germination percentage of transgenic seeds under 0 day and 6 days of AA is more compared to wild type Tellahamsa seeds, $\mathbf{d}$ AKR1 enzyme activity in rice seeds under control and ageing conditions. The total protein was extracted and enzyme activity was determined against Methylglyoxal as substrate. Minimum of three biological replicates were used in all the assays 

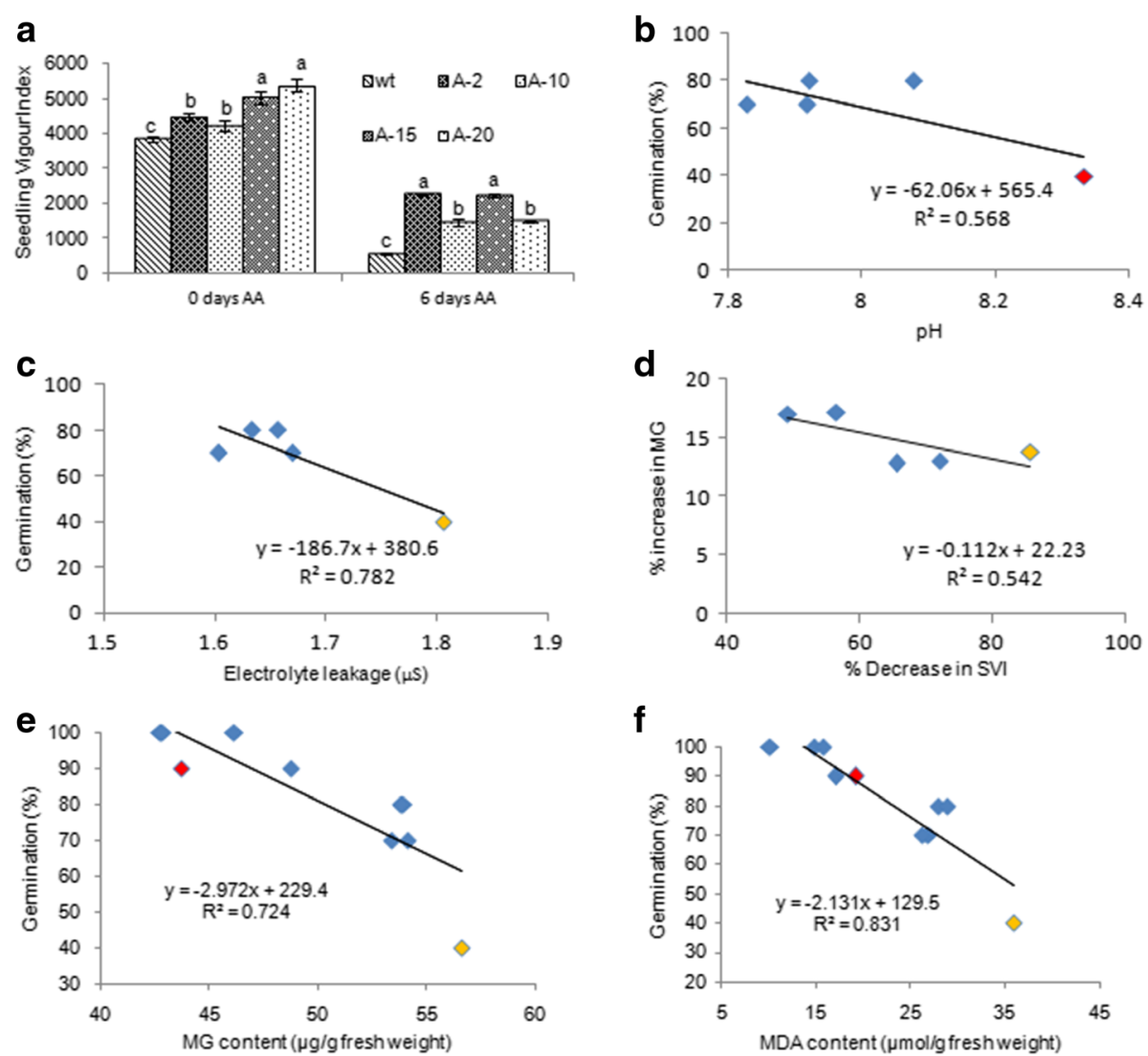

Fig. 5 Overexpression of PSAKR1 in Tellahamsa genotype rescued seed viability by scavenging cytotoxic compounds. a Transgenic seeds showed higher seedling vigour index compared to wild type Tellahamsa seeds, different letters above the error bars indicate significance levels, $n=10$, three biological replications with minimum 10 seeds were tested for ageing treatments. Student $t$-test for both wild type and transgenic seeds were conducted. Alphabets with same letters are non-significant at $p=<0.05$. b and $\mathbf{c}$ Correlation between germination percentage with electrolyte conductivity (EC) and pH of the seed leachate of rice transgenic and wild type seeds after soaking in water. $\mathbf{d}$ Correlation between reduction in methylglyoxal (MG) with seedling vigour index (SVI). e and $\mathbf{f}$ Correlation between germination with MG content and malondialdehyde (MDA) content. Data points in different colours indicate wild type seeds exposed to control (red), $48 \mathrm{~h}$ (yellow) and $72 \mathrm{~h}$ (green) of aging conditions

Managing the levels of RCCs is crucial to sustain the seed viability. From this context, RCC detoxifying enzymes assumes greater significance. The null rice mutants of Aldehyde dehydrogenase (ALDH7) showed increased MDA resulting in reduced seed viability (Shin and Kim 2009). The importance of AKR's in scavenging RCC has been shown in several other studies related to stress response. The reduced RCCs in rice, Arabidopsis and Alfalfa by overexpression of Aldo-keto reductase family genes showed increased tolerance to salinity, drought and heavy metals. The E. coli expressing OsAKR1 showed abiotic stress tolerance by detoxifying MG (Turoczy et al., 2011). Constitutive expression of Medicago sativa ALR1 in tobacco plants imparted tolerance to hydrogen peroxide, paraquat, salt and dehydration stresses (Oberschall, et al., 2000; Bartels, 2001). The detoxification of reactive carbonyl glyphosate by overexpression of PSAKR1 and OsAKR1 in tobacco improved tolerance against herbicide (Vemanna et al., 2016). The overexpression of PSAKR1 in tobacco showed enhanced protection of cellular enzymes from the salinity induced RCCs (Vemanna et al., 2017). From this context, increased seed longevity could be associated with the detoxification of carbonyl compounds that are substrates for Aldo-keto reductases. The transgenic tobacco and rice seeds expressing PSAKR1 showed reduced accumulation of RCCs like MDA, MG, Maillard products and Amadori compounds. The AKR activity in transgenics is correlated with the levels of MG and enhanced shoot growth of transgenics on MG further signifies that AKRs potentially detoxify these cytotoxic compounds. This resulted in reduced seed deterioration and enhanced viability of seeds when exposed to ageing treatment. The reduced levels of lipid peroxidation derived aldehydes found in the transgenic lines of tobacco and rice signifies the potential of $A K R 1$. This shows that $A K R 1$ expressing transgenic plants had improved detoxification ability of RCCs. 

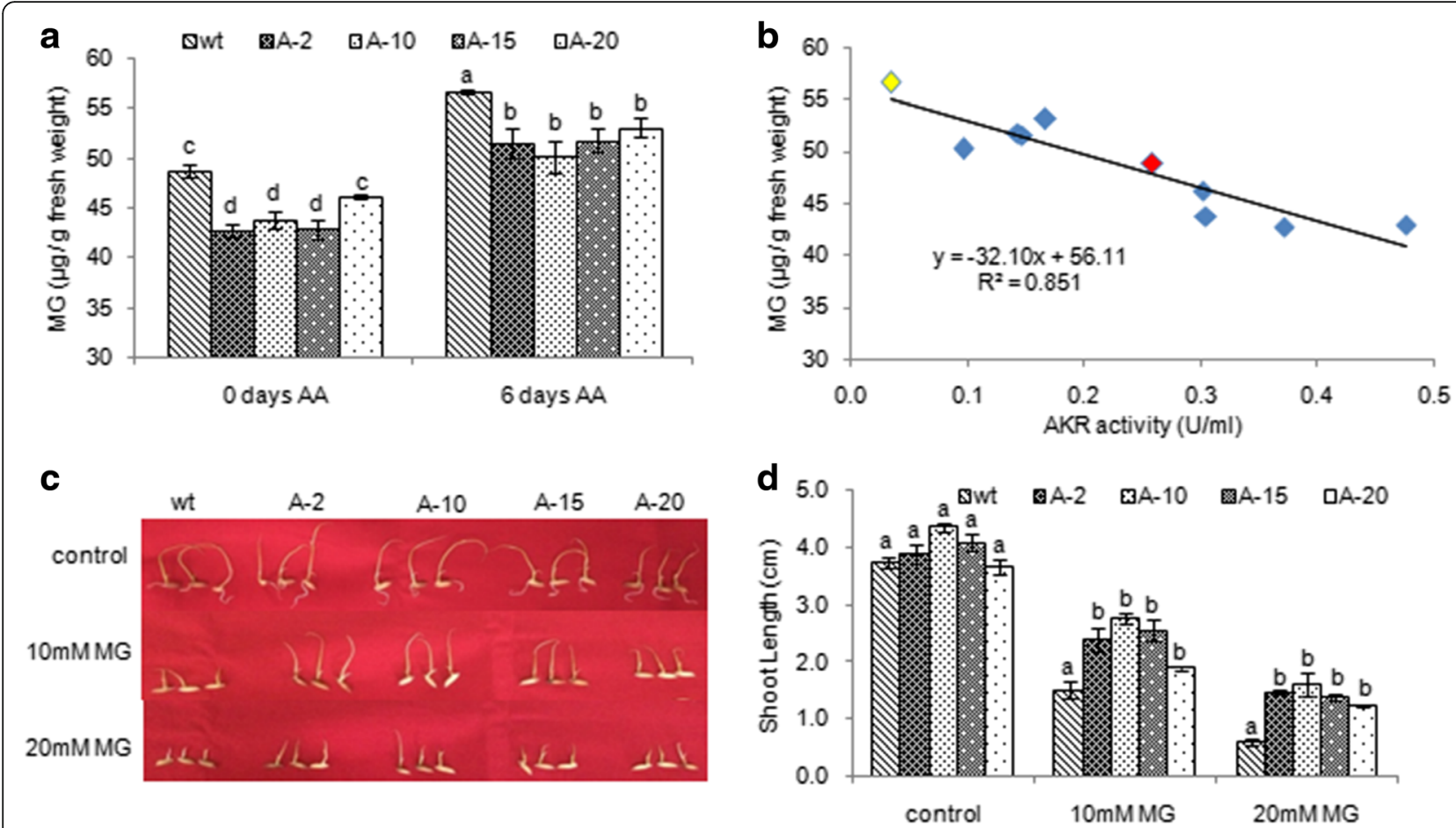

Fig. 6 The rice transgenics expressing PSAKR1 gene showed reduced methylgloxal (MG) levels in seeds under ageing conditions. a MG levels in transgenics and wild type seedlings under control and 6 days of accelerated ageing, $\mathbf{b}$ Correlation between reduction in MG with increased AKR activity, c Response of wild type and transgenic seedlings to $10 \mathrm{mM}$ and $20 \mathrm{mM} \mathrm{MG}$ after 4 days of treatment, $\mathbf{d}$ Shoot length of wild type and transgenics seedlings on MG treatment, $n=10$. Red and yellow dots represent values for wild type in control and ageing conditions respectively

\section{Conclusion}

In this study, the relevance of reactive cytotoxic compounds was assessed in rice genotypes by exposing to accelerated aging conditions. Under aging treatment the reactive cytotoxic compounds accumulated in all the genotypes and affect the seed viability. The genotypic variability is correlated with the accumulation of MDA, MG and Maillard products. Further, we characterize an Aldo-keto reductase (AKR) by developing transgenics in tobacco and rice susceptible genotype Telahamsa. The transgenics seeds showed improved AKR activity, viability and germination when exposed to the ageing treatments. The reduced levels of RCCs MDA, MG, Maillard and Amodari compounds in transgenics signifies that RCCs are detoxified by the AKRs. The recovery in seed viability in highly susceptible rice genotype Tellahamsa by overexpression of AKR1 clearly suggests that, detoxification of cytotoxic compounds by Aldo-keto reductases play a crucial role in maintaining the seed longevity and seedling vigour. The study demonstrates that, efficient detoxification of RCC's by overexpression of $A K R 1$ enhances the life span of seeds.

\section{Methods}

\section{Plant material}

Freshly harvested seeds of rice genotypes AC39020, KMP175, MAS, Tellahamsa, AC39018, AC35310, AC35313 and IET-16348 with known physiological and stress tolerant traits were used in the present study. To develop transgenic plants overexpressing PSAKR1 driven by ribulose 1,5-bis phosphate carboxylase (RBCS) promoter in pBINplus binary vector, rice genotype Tellahamsa and tobacco variety KST-19 were used. The Agrobacterium mediated in-planta transformation protocol for rice (Babitha 2012) and tissue culture based transformation protocol for tobacco was followed to develop transgenic plants. For rice inplanta transformation pre-germinated seed embryonic axis was wounded with a fine needle and the PSAKR1 construct harboring agrobacterium was infected. Since AKRs detoxify glyphosate, the promising rice transgenics were identified by screening on glyphosate in quartz sand as described in our previous study (Vemanna et al., 2016). The homozygous $\mathrm{T} 2$ generation seeds were used to assess the seed viability and vigour. Total genomic DNA was isolated from seedlings of wild type and transformed plants following the CTAB method (Dellaporta et al., 1983). PCR analysis was performed to amplify AKR1 gene using the primers (For primers, please see Additional file 1: Table S1) and the products were electrophoresed on a $1 \%$ agarose gel.

The total RNA was isolated according to Datta et al., (1989) to confirm the PSAKR1 expression in transgenic tobacco and rice seeds. The cDNA was synthesized 
by oligo (dT) primers using Moloney murine leukemia virus reverse transcriptase (MMLV-RT; MBI Fermentas, Bangalore). The qRT-PCR analysis was done using cDNA pool as a template. The gene specific primers were used to confirm the expression levels by qRT-PCR. The relative expression level of $A K R 1$ was calculated using the Image J software based on the band intensity. Actin was used as internal control for normalization (for primer, please see Additional file 1: Table S1).

\section{Accelerated ageing/controlled deterioration of seeds}

Dry seeds of rice genotypes were subjected to a standardized accelerated ageing treatment of $45{ }^{\circ} \mathrm{C}$ with $100 \%$ relative humidity for 6 days. Similarly, transgenic rice and tobacco seeds overexpressing PSAKR1 were also subjected to ageing treatment for 6 days and 3 days respectively. Seeds in paper covers were placed inside desiccators with water to maintain $100 \% \mathrm{RH}$ and were kept inside incubator (Delouche and Baskin 1973). After 3 and 6 days of incubation, seeds were removed from the desiccators and subjected to germination assay.

\section{Methyl glyoxal treatment assay}

Transgenic and wild type seeds were pre-germinated and placed on agar plates with half MS media supplemented with $10 \mathrm{mM}$ and $20 \mathrm{mM} \mathrm{MG}$. After 4 days the shoot length of the seedlings was recorded in both the treatments.

\section{Germination and seedling growth}

The seeds removed from the accelerated ageing treatment and from control conditions were imbibed for $4 \mathrm{~h}$ and then placed in petri plates with moistened blotting paper. Ten seeds from each genotype were used for the study with three replications. After two days, the percent seed germination was measured and later, root length and shoot length was also measured. Based on the data generated, germination speed index (GSI) was calculated (AOSA, 1983) with some modifications. GSI $=($ Total no. of seeds sown/initial germinated seeds) + (Total no. of seeds sown/ final germinated seeds). In addition to GSI, seedling vigour index was also calculated using the formula, SVI $=$ Germination percentage $\mathrm{x}$ (root length + shoot length) (Abdul-Baki and Anderson, 1973).

\section{$\mathrm{pH}$ of the seed leachate media}

Both accelerated aged and control seeds were soaked in a known volume of water overnight and the $\mathrm{pH}$ of the seed leachate was measured using digital $\mathrm{pH}$ meter (Systronics, $\mu \mathrm{pH}$ system 361, Delhi, India).

\section{Electrolyte leakage in the media}

After accelerated ageing treatment, the seeds were soaked in a known volume of water overnight and the electrolyte leakage (EC) from seed leachate was measured using electrical conductivity meter (Elico, CM180, Hyderabad, India) and expressed in micro Siemens $(\mu S)$. Initial EC was measured in water before soaking the seeds and then seeds were soaked in water overnight and final EC was measured from the leachate. The electrolyte leakage was calculated using the following formula: EC (\%) = (initial EC/final EC) $\times 100$.

\section{Assessment of RCC's upon ageing treatment}

Reactive carbonyl compounds like MDA, MG, Amadori and Maillard products were quantified in rice genotypes as well as in tobacco and rice transgenics overexpressing PsAKR1 gene.

\section{MDA}

About 0.5-1.0 gram of seeds exposed to ageing treatment was homogenized in $5 \mathrm{ml}$ of $10 \%$ (W/V) trichloroacetic acid (HiMedia, Nasik, Maharashtra) and $0.25 \%$ of thiobarbutiric acid. The homogenate was centrifuged at 12,000 rpm for $15 \mathrm{~min}$ at room temperature. The supernatant was mixed with an equal amount of thiobarbutiric acid $[0.5 \%$ in $20 \%(\mathrm{~W} / \mathrm{V})$ trichloroacetic acid] (Sigma aldrich, Bangalore, India) and the mixture was boiled for $25 \mathrm{~min}$ at $100{ }^{\circ} \mathrm{C}$ followed by centrifugation for $5 \mathrm{~min}$ at $7,500 \mathrm{rpm}$ to clarify the solution. Absorbance of the supernatant was measured at $532 \mathrm{~nm}$ and $600 \mathrm{~nm}$ and corrected for nonspecific turbidity by subtracting the absorbance at A600. The standard MDA (Sigma Aldrich, Bangalore, India) was used to develop the standard graph.

\section{Methyl glyoxal (MG)}

MG was quantified in aged and control seeds according to Yadav et al., (2005). One hundred mg of tissue was taken and ground in a known volume of distilled water and centrifuged at $11,000 \mathrm{rpm}$ for $10 \mathrm{~min}$ at $40 \mathrm{C}$ and supernatant was collected. To quantify the MG content, $250 \mu \mathrm{l}$ of $7.2 \mathrm{mM}$ of 1,2-diamino benzene (1,2-phenylenediamine), $100 \mu \mathrm{l}$ of $5 \mathrm{M}$ perchloric acid and $650 \mu \mathrm{l}$ of the neutralized supernatant were added. The absorbance was read at $336 \mathrm{~nm}$ using spectrophotometer (Spectra max plus-384, Spinco Biotech pvt ltd, Bangalore).

\section{Amadori and Maillard products}

One hundred mg seeds of both control and aged seeds were ground in $1.2 \mathrm{ml}$ of $50 \mathrm{mM}$ phosphate buffer ( $\mathrm{pH}$ 7.2). The homogenate was vortexed and centrifuged at 12,000 rpm for $15 \mathrm{~min}$. Further, ammonium sulphate of $0.5 \mathrm{~g} \mathrm{ml}-1$ was added to precipitate the proteins. The pellet was dissolved in $3.3 \mathrm{ml}$ phosphate buffer $(50 \mathrm{mM}$, $\mathrm{pH}$ 7.2). Extracted proteins were used to measure the Amadori reaction products and Maillard reaction products. The Amadori reaction products were measured using the nitro-blue tetrazolium (NBT) method (Wettlaufer and 
Leopold, 1991). To this, $1 \mathrm{ml}$ of NBT reagent $(0.5 \mathrm{mM}$ NBT in $100 \mathrm{mM}$ sodium carbonate, $\mathrm{pH}$ 10.3) was added to $0.2 \mathrm{mg}$ of extracted proteins and incubated at $400 \mathrm{C}$ in a water bath. The absorbance at $550 \mathrm{~nm}$ was recorded after 10 and 20 min of incubation using spectrophotometer.

The content of Maillard reaction products was determined using the fluorescence method using flurometer (Fluromax, Jobinyvonhoriba, USA). Extracted seed proteins (0.3 mg ml-1) were scanned with an excitation wavelength from $270-400 \mathrm{~nm}$ and emission wavelengths from $320-500 \mathrm{~nm}$. A new fluorescence maximum was detected at the excitation of $350 \mathrm{~nm}$ and emission of $420 \mathrm{~nm}$ (Murthy and Sun, 2000). The intensity of the new fluorescence peak was used to express the content of Maillard reaction products in seed proteins. Proteins were quantified and BSA was used to develop standard graph.

\section{Alpha amylase activity}

Amylase activity was determined by DNS (Di-nitro-salicyclic acid) method (Bernfeld, 1955). Glucose solution of $400 \mu \mathrm{g} / \mathrm{ml}$ and $1 \%$ starch solution was used as standard, One gram seed from both control and aged treatment was weighed and incubated in water for a known period of time and later homogenized with $9 \mathrm{ml}$ of phosphate buffer ( $\mathrm{pH}-7.5)$. The homogenate was centrifuged at 3,000 rpm for $10 \mathrm{~min}$. To measure the enzyme activity $1.5 \mathrm{ml}$ of 0.1 M phosphate buffer ( $\mathrm{pH}-7.5$ ) was added and the mixture was incubated at room temperature for $5 \mathrm{~min}$ and then added the enzyme mix. The reaction was allowed to continue for $20 \mathrm{~min}$ and was stopped by the addition of $2 \mathrm{ml}$ DNS reagent. The test tubes were placed in a boiling water bath for $10 \mathrm{~min}$ and cooled. The optical density of the orange yellow coloured complex was measured at $540 \mathrm{~nm}$ and the values were recorded. The extent of reducing sugar formed (an indication of starch hydrolysis due to the action of amylase activity) was determined by reading the OD values against glucose standard graph.

\section{Total sugar content estimation}

Anthrone method was followed to quantify the total sugars. One gram seed tissue from both control and aged treatment was immersed separately in ethanol and allowed to boil for $10 \mathrm{~min}$. The tissue was ground and filtered until complete removal of alcohol soluble substances. The extracts were then centrifuged at $8000 \mathrm{rpm}$ and later made up the volume to $10 \mathrm{ml}$ with distilled water. One $\mathrm{ml}$ of aliquot was pipette out and made up to the volume to $2.5 \mathrm{ml}$ with distilled water and added $5 \mathrm{ml}$ of Anthrone reagent slowly. Then the reaction mixture was heated on boiling water bath exactly for $7.5 \mathrm{~min}$ and cooled immediately in ice bath. After cooling, the absorbance of the solutions at $630 \mathrm{~nm}$ was measured. The sugar content was calculated through standard glucose curve (Loewus, 1952).

\section{Measurement of TTC activity}

Hulled seeds of both control and aged treatment were pre-conditioned by soaking in distilled water at $28{ }^{\circ} \mathrm{C}$ for $4 \mathrm{~h}$ and transferred them in 1\% tetrazolium chloride solution for $6 \mathrm{~h}$ at room temperature in dark, and then washed several times with distilled water to remove excess solution. Two hundred mg of seeds incubated in TTC solution was ground in $1 \mathrm{ml}$ of SDS and centrifuged at $8,000 \mathrm{rpm}$ for $20 \mathrm{~min}$. Later, the supernatant was collected and the extent of colour development was assessed based on $\mathrm{OD}$ values at $485 \mathrm{~nm}$ in spectrophotometer.

\section{Measurement of AKR activity}

The AKR activity in the seeds was measured using MG as substrate. The total protein from $0.4 \mathrm{~g}$ of seeds was extracted in $400 \mu \mathrm{l}$ of isolation buffer $[25 \mathrm{mM}$ Tris$\mathrm{HCl} \mathrm{pH} \mathrm{7.6,} 15 \mathrm{mM} \mathrm{MgCl} 215 \mathrm{mM}$ ethylene glycol tetraacetic acid, $75 \mathrm{mM} \mathrm{NaCl}, 60 \mathrm{mM}$ b-glycerophosphate, 2 mM 1,4-DithioL-threitol, 0.1\% Igepal CA-630 (SigmaAldrich), $1 \mathrm{mM} \mathrm{NaF}, 1 \mathrm{mM}$ phenylmethanesulfonylfluoride (Sigma-Aldrich)]. The homogenate was centrifuged for $10 \mathrm{~min}$ at 13,000 $\mathrm{g}$ and the supernatant was saturated using $25 \%$ ammonium sulphate. The proteins precipitated were dissolved in small amount of $0.1 \mathrm{M}$ sodium phosphate buffer, $\mathrm{pH}$ 7. All steps were done at $0-4{ }^{\circ} \mathrm{C}$. Total protein content is quantified using Bradford's method. The AKR activity was determined using $1 \mu \mathrm{g}$ of crude protein and $0.1 \mathrm{mM}$ NADPH and $2 \mathrm{mM}$ Methyl glyoxal substrate (Sigma-Aldrich). The absorbance at $340 \mathrm{~nm}$ at $25{ }^{\circ} \mathrm{C}$ to monitor decrease in $\mathrm{NADPH}$ was quantified using spectrophotometer (Spectra max plus384, Spinco Biotech pvt ltd, Bangalore) and AKR activity was calculated using the molar extinction co-efficient (0.623) (Turcozy et al., 2011).

\section{Additional file}

Additional file 1: Table S1. List of Primers used in the study. Figure S1. Differential accumulation of RCC's affected germination after ageing treatments in rice genotypes determined genotypic variation. Figure S2. Accumulation of different reactive carbonyl compounds in transgenics tobacco seeds expressing PSAKR1. Figure S3. Overexpression of PSAKR1 in susceptible genotype Tellahamsa showed reduced malondialdehyde and less electrolyte leakage. (PDF 1708 kb)

\footnotetext{
Acknowledgements

This work was supported from Department of Biotechnology, Centre of Excellence program support (BT/01/COE/05/03), Indian Council of

Agricultural Research - Niche Area of Excellence program (F. No. 10-(6)/2005 EPD) and (F. No. 10 (15) 2012 EPD), Department of Science and Technology Fund for Improvement of Science and Technology (SR/FST/LSI-051/2002). MUK acknowledges the support for Platinum jubilee fellowship from NASI, India. K. N.N. acknowledge the fellowship from Department of Biotechnology-University of Agricultural Sciences, Biotech HUB (Ref No. BT/INF/UAS, Bangalore/2011). Lakshmi and Manjunath S for lab help.
} 


\section{Authors' contributions}

UM, RSV \& MRB conceived the study and coordinated the project. NKN did accelerated ageing experiments. RSV, NKN, BKC analyzed the data and wrote manuscript. HR developed transgenic tobacco plants. ARV did seed viability assay and AN developed rice transgenic plants. UM, RSV, NKN \& MR wrote manuscript. All authors read and approved the final manuscript.

\section{Competing interests}

The authors declare that they have no competing interests.

\section{Publisher's Note}

Springer Nature remains neutral with regard to jurisdictional claims in published maps and institutional affiliations.

\section{Received: 24 November 2016 Accepted: 17 March 2017}

\section{Published online: 13 April 2017}

\section{References}

Abdul-Baki AA, Anderson JD (1973) Vigour determination in soybean by multiple criteria. Crop Sci 10:31-34

Andreuzza S, Li J, Guitton A, Faure JE, Casanova S, Park JS, Choi Y, Chen Z, Berger F (2010) DNA ligase I exerts a maternal effect on seed development in Arabidopsis thaliana. Development 137:33-81

Association of Official Seed Analysts (1983) Seed Vigor Testing Handbook. Edited by Drs. Riad Baalbaki, Sabry Elias, Julio Marcos-Filho, and Miller B. McDonald. Contribution No. 32. 89pp.

Babitha KC (2012) Development of multiple gene construct with regulatory genes and their functional validation. PhD Thesis. University of Agricultural sciences, GKVK, Bangalore

Bartels D (2001) Targeting detoxification pathways: an efficient approach to obtain plants with multiple stress tolerance? Trends Plant Sci 6:284-6

Bernfeld P (1955) Amylases, $a$ and $\beta$. Methods Enzymol 1:149-158

Bueso E, Ibanez C, Sayas E, Bertomeu JM, Guzman MG, Pedro L, Rodriguez SR (2014) A forward genetic approach in Arabidopsis thaliana identifies a RINGtype ubiquitin ligase as a novel determinant of seed longevity. Plant Science 215:110-116

Colrat S, Latche A, Guis M, Pech JC, Bouzayen M, Fallot J, Roustan JP (1999) Purification and characterization of a NADPH-dependent aldehyde reductase from mung bean that detoxifies eutypine, a toxin from Eutypa lata. Plant Physiol 119:621-626

Datta K, Schmiit A, Marces A (1989) Characterization of two Soybean repetitive proline rich proteins and a cognate cDNA from germinated axes. Plant Cell 1:945-952

Dellaporta SL, Wood J, Hicks JB (1983) A plant DNA minipreparation: version II. Plant Mol Biol Rep 1:19-21

Delouche JC, Baskin CC (1973) Accelerated aging techniques for predicting the relative storability of seed lots. Seed Sci Technol 1:427-452

Feussner I, Wasternack C (2002) The lipoxygenase pathway. Annu Rev Plant Bio 53:275-297

Gidrol X, Serghini H, Noubhani A, Mocquot B, Mazliak P (1989) Biochemical changes induced by accelerated aging in sunflower seeds. 1. Lipid peroxidation and membrane damage. Physiol Plant 76:591-597

Hasanuzzaman M (2015) Concept note. http://hasanuzzaman.weebly.com/ uploads/9/3/4/0/934025/seed_quality.pdf.

Kapoor N, Arya A, Siddiqui MA, Kumar H, Amir A (2011) Physiological and biochemical changes during seed deterioration in aged seeds of Rice. Am J Plant Physiol 6:28-35

Kibinza S, Vinel D, Come D, Bailly C, Corbineau F (2006) Sunflower seed deterioration as related to moisture content during ageing, energy metabolism and active oxygen species scavenging. Physiol Plant 128:496-506

Koning RE (1994) Seeds and seed germination. Plant physiology information website. http://plantphy.info/plants_human/seedgerm.s/html.

Loewus FA (1952) Improvement in Anthrone Method for Determination of Carbohydrates. Anal Chem 24:219-219

Mano J (2012) Reactive carbonyl species: Their production from lipid peroxides, action in environmental stress, and the detoxification mechanism. Sci Res Center 45:1-8

Miyata T, Kurokawa K, Strihou V (2000) Molecular and Cellular Nephrology, Institute of Medical Advanced Glycation and Lipoxidation End Products: Role of Reactive Carbonyl Compounds Generated during Carbohydrate and Lipid Metabolism. J Am Soc Nephrol 11:1744-1752
Mohammadi H, Soltani A, Sadeghipour HR, Zeinali E (2011) Effect of seed aging on subsequent seed reserve utilization and seedling growth in soybean. Inter Jour Plant Production 5:65-70

Mudgett MB, Clarke S (1993) Characterization of plant L-isoaspartyl methyltransferases that may be involved in seed survival: Purification, cloning, and sequence analysis of the wheat germ enzyme. Biochemistry 32:11100-11111

Mudgett MB, Lowenson JD, Clarke S (1997) Protein repair L-isoaspartyl methyltransferase in plants. Phylogenetic distribution and the accumulation of substrate proteins in aged barley seeds. Plant Physiol 115:1481-1489

Murthy UMN, Sun WQ (2000) Protein modification by the Amadori and Maillard reactions during seed storage: roles of sugar hydrolysis and lipid peroxidation. J Exp Bot 51:1221-1228

Murthy N, Prakash UM, Kumar P, Wendell Q (2003) Mechanisms of seed ageing under different storage conditions for Vigna radiata (L.)lipid peroxidation, sugar hydrolysis, Maillard reactions and their relationship to glass state transition. J Exp Bot 54:1057-1067

Oberschall A, Deak M, Sass L, Vass I, Kovacs A, Feher Dudits G, Horvath L (2000) A novel aldose/aldehyde reductase protects transgenic plants against lipid peroxidation under chemical and drought stresses. Plant J 24:437-446

Oge L, Bourdais G, Bove J, Collet B, Grappin P (2008) Protein repair L-isopaspartyl methyltransferase 1 is involved in both seed longevity and germination vigour in Arabidopsis. Plant Cell 20:3022-3037

Ott C, Jacobs K, Haucke E, Santos AN, Grune T, Simm A (2014) Role of advanced glycation end products in cellular signaling. Redox Biol 2:411-429

Rao K, Jackson (1996) Seed longevity of rice cultivars and strategies for their conservation in genebanks. Ann Bot 77:251-260

Richard JP (1984) Acid-base catalysis of the elimination and isomerization reactions of triose phosphates. J A Chem Soc 106:4926-4936

Sayre LM, Lin D, Yuan Q, Zhu X, Tang X (2006) Protein adducts generated from products of lipid peroxidation: focus on HNE and ONE. Drug Metab Rev 38:651-675

Semchyshyn HM (2014) Reactive Carbonyl Species In vivo: Generation and Dual Biological Effects. Scientific World J 2014: 4178421-10.

Shaban M (2013) Review on physiological aspects of seed deterioration. Intl J Agri Crop Sci 6:627-631

Shin JH, Kim SR, An G (2009) Rice Aldehyde Dehydrogenase 7 is needed for seed maturation and viability. Plant Physiol 149:905-915

Sree BK, Rajendrakumar SV, Reddy AR (2000) Aldose reductase in rice (Oryza sativa L.): stress response and developmental specificity. Plant Sci 160:149-157

Sun WQ, Leopold AC (1995) The Maillard reaction and oxidative stress during ageing of soybean seeds. Physiol Plant 94:94-105

Takagi D, Inoue H, Odawara M, Shimakawa G, Miyake C (2014) The Calvin cycle inevitably produces sugar-derived reactive carbonyl methylglyoxal during photosynthesis: a potential cause of plant diabetes. Plant Cell Physiol 55:333-340

Turoczy Z, Kis P, Torok M, Lendvai D, Dudits-Horváth GV (2011) Overproduction of a rice aldo-keto reductase increases oxidative and heat stress tolerance by malondialdehyde and methylglyoxal detoxification. Plant Mol Bio 75:399-412

Vemanna SR, Amarnatha Reddy V, Murugesh E, Babitha KC, Hanumantha R, Kirankumar G, Chinta S, Kirankumar SM, Udayakumar M (2016) Aldoketoreductase enzymes detoxify glyphosate and improve herbicide resistance in plants. Plant Biotechnol, doi:10.1111/pbi.12632

Vemanna SR, Babitha KC, Solanki JK, Amarnatha Reddy V, Sarangi SK, Udayakumar M (2017) Aldo-keto reductase-1 (AKR1) protect cellular enzymes from salt stress by detoxifying reactive cytotoxic compounds. Physiol Biochem 113:177-186

Ventura L, Dona M, Macovei A, Carbonera D, Buttafava A, Mondoni A (2012) Understanding the molecular pathways associated with seed vigor. Plant Physiol Biochem 60:196-206

Wang F, Wang R, Jing W, Zhang W (2012) Quantitative dissection of lipid degradation in rice seeds during accelerated aging. Plant Growth Regul 66:49-58

Wettlaufer SH, Leopold AC (1991) Relevance of Amadori and Maillard products to seed deterioration. Plant Physiol 97:165-169

Yadav SK, Singla-pareek SL, Ray M, Reddy MK, Sopory SK (2005) Methylglyoxal levels in plants under salinity stress are dependent on glyoxalase I and glutathione. Biochem Biophys Res Commun 337:61-67

Zhou Y, Chu P, Chen H, Li Y, Liu J, Ding Y, Tsang EW, Jang LL, Wu K, Huang SS (2012) Overexpression of Nelumbo nucifera metallothionens 2a and 3 enhances seed germination vigor in Arabidopsis. Planta 235:523-537 